\title{
Intranasal challenge with aspirin in the diagnosis of aspirin intolerant asthma: evaluation of nasal response by acoustic rhinometry
}

\author{
J Casadevall, P-J Ventura, J Mullol, C Picado
}

Servei de Pneumologia Hospital General, Vic, Spain

J Casadevall

Unitat

d'Epidemiologia i

Bioestadistica, Institut d'Investigacions Bimédiques August Pi

i Sunyer (IDIBAPS),

Barcelona, Spain

P-J Ventura

Institut d'

Investigacions

Bimédiques August Pi

i Sunyer (IDIBAPS)

Barcelona, Spain

P-J Ventura

J Mullol

C Picado

Servei de

Otorrinolaringologia, Hospital Clinic, Barcelona, Spain

J Mullol

Servei de

Pneumologia, Institut Clinic de Pneumologia i Cirurgia Toràcica, Hospital Clinic, Departament de Medicina, Facultat de Medicina, Barcelona, Spain

C Picado

Correspondence to: Dr C Picado, Institut de Pneumologia, Villarroel 170, Hospital Clinic, 08036 Barcelona, Spain cpicado@medicina.ub.es

Received 9 March 2000 Returned to authors 23 June 2000 Revised manuscript received 19 July 2000

Accepted for publication

8 August 2000

\begin{abstract}
Background-Nasal provocation tests with lysine-aspirin have recently been introduced for assessment of aspirin intolerant asthma. A study was undertaken to evaluate the usefulness of acoustic rhinometry, a new non-invasive technique, in the diagnosis of aspirin intolerant asthma/rhinitis.
\end{abstract}

Methods-Fifteen patients with aspirin intolerant asthma/rhinitis (nine women, mean (SD) age 54.7 (14) years), eight patients with aspirin tolerant asthmal rhinitis (three women, mean (SD) age 52.6 (7.8) years), and eight healthy subjects (two women, mean (SD) age 32.5 (9.7) years) were studied. All subjects were challenged with saline $(0.9 \% \mathrm{NaCl})$ and $25 \mathrm{mg}$ lysine acetylsalicylic acid (L-ASA) instilled into each nostril of the nose on two separate days. The clinical response was evaluated based on nasal symptoms (sneezes, itching, secretion and blockage). The nasal response was measured by acoustic rhinometry. Symptoms and rhinometry curves were recorded at $\mathbf{1 0}$ minute intervals for three hours, one hour before challenge and two hours after challenge.

Results-L-ASA challenge induced a significant increase in symptoms in patients with aspirin intolerant asthma/rhinitis. No differences in the clinical response were detected in those with aspirin tolerant asthma/rhinitis or healthy subjects. L-ASA challenge induced a significant decrease in nasal volume measured by acoustic rhinometry in aspirin intolerant patients. No differences were detected between the challenges in aspirin tolerant patients. If a $25 \%$ decrease in nasal volume is taken as the cut off point, the specificity of the test was $94 \%$ and the sensitivity reached $73 \%$. The nasal challenge was well tolerated by all subjects.

Conclusion-Acoustic rhinometry may be used to study the nasal response to L-ASA. Nasal challenge with L-ASA is safe and can be used as a diagnostic test even in asthmatic patients with severe bronchial obstruction.

(Thorax 2000;55:921-924)

Keywords: aspirin sensitive asthma; rhinitis; nasal provocation test; acoustic rhinometry
The Widal triad is a clinical syndrome characterised by the presence in the same patient of bronchial asthma, aspirin sensitivity, and chronic rhinosinusitis with nasal polyposis. These patients frequently suffer from a severe, often steroid dependent, form of bronchial asthma but they also have chronic rhinosinusitis which is difficult to manage, complicated by recurrent nasal polyposis. ${ }^{1}$

There is substantial evidence to support the notion that the processes affecting the nose and the lower airways share common pathogenic mechanisms. Increased numbers of activated eosinophils, mast cells, and $\mathrm{T}$ lymphocytes are usually found in these conditions. ${ }^{23}$ The basic mechanism underlying sensitivity to aspirin involves inhibition of cyclo-oxygenase with subsequent release of cysteinyl leukotrienes in the lower and upper airways. ${ }^{4-6}$ The phenomenon of sensitivity to aspirin (acetylsalicylic acid, ASA) provides additional evidence for common pathogenic mechanisms in ASA induced rhinitis and ASA induced asthma.

Oral ASA challenge induces both bronchial and nasal reactions in most patients with ASA intolerant asthma/rhinitis. Direct challenge of either the airways or the nose with lysine-ASA (L-ASA) induces an increase in cysteinyl leukotriene production. ${ }^{4-6}$

Oral ASA and inhaled L-ASA challenges are commonly used to investigate aspirin intolerant asthma for diagnostic and research purposes. ${ }^{7}$ The main limitation of the oral test is the possibility of inducing severe systemic and bronchospastic responses. The inhaled test is better tolerated and the only risk derives from excessive bronchospasm. For safety reasons, neither test can be used in patients with moderate or severe bronchial obstruction (patients must have a forced expiratory volume in one second $\left(\mathrm{FEV}_{1}\right)$ of $65 \%$ or higher than predicted). ${ }^{7}$ In contrast, the nose can be challenged repeatedly without any apparent risk, even in patients with severe bronchial obstruction. ${ }^{8}$ Patriarca et $a l^{9}$ were the first to use the aspirin nasal challenge for diagnostic purposes. Although there are arguments in favour of the use of nasal challenge to investigate ASA intolerance, the usefulness of this method in the diagnosis of this syndrome has only recently been investigated using rhinomanometry. Rhinomanometric measurement requires the generation of nasal flow. The presence of nasal polyps in aspirin sensitive patients usually makes it difficult to generate nasal flow, especially when nasal obstruction increases after the instillation of L-ASA. 
Acoustic rhinometry is a non-invasive technique in which the nasal geometry is assessed by means of a reflected sound. The experimental equipment needed for the acoustic reflection measurement of nasal airways volume includes a trigger module to produce the acoustic pulse, a wave tube, a piezoelectric microphone, an amplifier, and an analogue/ digital converter. This method involves the measurement of acoustic reflections from the nasal cavity of a sound pulse created by a spark in a sound tube connected to the nasal cavity via a nosepiece. The results are presented as a curve describing the cross sectional area of the nasal cavity as a function of the distance from the nostril. ${ }^{10}$ This technique has been applied with good results in otorhinolaryngological conditions ${ }^{11-13}$ where it has been shown to have better repeatability and validity than classical rhinomanometry. ${ }^{14}$ Unlike rhinomanometry, acoustic rhinometry does not require generation of nasal flow and therefore its use is less limited by the presence of nasal polyps and/or nasal obstruction. These drawbacks meant that Milewski et $a l^{8}$ could not perform rhinomanometric evaluation in $20 \%$ of their patients with aspirin intolerant asthma/rhinitis.

Our study aimed to determine the usefulness of acoustic rhinometry in the diagnosis of aspirin intolerance. Our main objective was to establish several cut off points in the percentage fall in the nasal volume and to evaluate their sensitivity and specificity in the diagnosis of aspirin sensitive asthma.

\section{Methods}

PATIENTS

Fifteen asthmatic patients with a clear cut history of aspirin intolerant asthma and rhinitis were included in the study. In eight patients who had only had one attack of asthma and rhinitis precipitated by aspirin or related drugs, aspirin sensitivity was confirmed by an oral ASA challenge according to the method described elsewhere. ${ }^{15}$ In seven patients with more than one attack precipitated by various non-steroidal inflammatory drugs, the oral challenge was not performed.

Eight patients with aspirin tolerant asthma and rhinitis challenged with $500 \mathrm{mg}$ ASA with negative results and eight healthy control subjects who were not orally challenged were also included.

The doses of nasal and oral corticosteroids were not changed during the course of the study.

All subjects gave informed consent to participate in the study which was approved by the local ethical committee.

NASAL CHALLENGE PROCEDURE

All subjects were challenged with saline $(0.9 \%$ $\mathrm{NaCl}$ ) and $25 \mathrm{mg} \mathrm{L}$-ASA instilled into each nostril of the nose. Nasal challenge was performed with the patient seated. The nasal response was evaluated by acoustic rhinometry and the clinical response by scoring the subjective nasal symptoms. The clinical symptoms score included rhinorrhoea, nasal blockage, itching of the nose, palate or throat, and sneez- ing. The intensity of symptoms was scored from $0=$ none to $10=$ very severe for rhinorrhoea and blockage, $0=$ absent and $1=$ present for itching and the number of sneezes. The sum of the individual scores was calculated as a composite nasal score.

Nasal symptoms and nasal volume were measured in the first 30 minutes at 10 minute intervals (period 1). Anterior rhinoscopy was performed to evaluate the presence or absence of nasal polyps and $80 \mu \mathrm{l} 0.9 \% \mathrm{NaCl}$ was then instilled into each inferior turbinate with an Eppendorf pipette and the symptoms and nasal volume were assessed at 10 minute intervals for an additional 30 minutes (period 2).

To perform the aspirin challenge $900 \mathrm{mg}$ L-ASA (Aspisol; Bayer, Germany) was diluted in $2.8 \mathrm{ml} 0.9 \% \mathrm{NaCl}$ and $80 \mu \mathrm{l}$ of this solution, representing $25 \mathrm{mg} \mathrm{L}-\mathrm{ASA}$, was applied to each inferior turbinate. Nasal symptoms and volumes were measured after L-ASA instillation at 10 minute intervals for two hours (period 3).

The placebo challenge was performed with the same protocol using the same volume of $0.9 \% \mathrm{NaCl}$.

The study was double blind and each patient was challenged with L-ASA and placebo. Both challenges were separated by at least one week and performed in a random order.

ACOUSTIC RHINOMETRY

A detailed description of the acoustic rhinometry technique can be found elsewhere. ${ }^{10}{ }^{11}$ We used an acoustic rhinometer (SRE2000PC, SR Electronics ApS, Lynge, Denmark). This technique makes it possible to obtain a curve of the cross sectional area at different positions in the nostril, and a software program enables the volume of each nostril, the minimal cross sectional area, and its distance from the nosepiece to be calculated. Rhinometric measurements were performed while the subject was in apnoea after a non-forced expiration. The volume of the nasal cavity was measured from the distal extreme of the nosepiece to $12 \mathrm{~cm}$ from the rear.

The rhinometer was calibrated daily with a calibration tube provided by the manufacturer.

\section{ANALYSIS OF DATA}

The primary variable studied was the variation in the total nasal volume obtained by the sum of the nasal volume measured in each nostril from which the percentage change in nasal volume $(\% \mathrm{NV})$ was calculated. During periods 1 and 2 the $\% \mathrm{NV}$ was obtained using the first nasal volume measurement (time 0 ) as the baseline value. During period 3 the $\% \mathrm{NV}$ was calculated by taking the nasal volume 30 minutes after instillation of saline as the baseline value (time 60).

Since the nasal volume and $\% \mathrm{NV}$ values were not normally distributed, a logarithmic transformation was applied to the data before formal analysis. Another variable studied was the change in the subjective composite symptom score at the same time intervals as the nasal volume. 
Table 1 Characteristics of study subjects

\begin{tabular}{llll}
\hline & AIAR $(n=15)$ & ATAR $(n=8)$ & Controls $(n=8)$ \\
\hline Mean (SD) age/range & $55(14) / 34-70$ & $53(8) / 41-64$ & $32(10) / 24-53$ \\
M:F & $6: 9$ & $5: 3$ & $6: 2$ \\
$\mathrm{FEV}_{1}(\%$ predicted)/range & $64(22) / 27-96$ & $81(12) / 58-99$ & $88(3) / 81-98$ \\
Intranasal corticosteroids & 14 & 8 & 0 \\
$\quad$ No of patients & $520 / 400-1000$ & $400 / 200-800$ & 0 \\
$\quad$ Dose $(\mu \mathrm{g} /$ day)/range & 4 & 1 & 0 \\
$\quad$ Oral prednisone & $8.3 / 5-15$ & 5 & 0 \\
$\quad$ No of patients & & & \\
\hline
\end{tabular}

$\mathrm{AIAR}=$ aspirin intolerant asthma/rhinitis; $\mathrm{ATAR}=$ aspirin tolerant asthma/rhinitis.

We initially performed a descriptive analysis of the baseline characteristics (age, sex, and presence of nasal polyps) for each group using the Student's $t$ test and $\chi^{2}$ test. Multivariate analysis (ANOVA of repeated measures) was used, including in the model both the variables distributed differently in the groups and the variables considered to be clinically relevant.

Statistical analysis was performed with a MLn program (Multilevel Models Project, Institute of Education, University of London, UK). The results were considered statistically significant if the $\mathrm{p}$ value was $\leqslant 0.05$.

\section{Results}

Thirty one subjects (14 women) of mean (SD) age 48 (15) years were studied. Fifteen subjects had aspirin intolerant asthma/rhinitis, eight had aspirin tolerant asthma/rhinitis, and there were eight healthy control subjects. Fourteen patients $(45 \%)$ had nasal polyposis. The baseline characteristics of the subjects are shown in table 1. The groups differed in age and presence of nasal polyps. The mean age of the healthy group was significantly lower than the other two groups. The mean baseline nasal volume was also significantly different between healthy subjects and patients. There was a tendency to a slight but non-significant decrease in the nasal volume during the first hour (periods 1 and 2) in the three groups (data not shown).

Figure 1 shows the $\% \mathrm{NV}$ after L-ASA and placebo in the three groups. There were no differences in the response to placebo between the three groups. In contrast, a significant decrease in the nasal volume was found from 50 minutes to the end of the study in patients with aspirin intolerant asthma/rhinitis compared with aspirin tolerant asthmatics and healthy subjects.

Intranasal challenge with L-ASA induced a significant increase in clinical symptoms, mainly nasal congestion and rhinorrhoea, 30 minutes after L-ASA instillation compared with saline in aspirin intolerant patients. No patient complained of itching. L-ASA did not induce sneezing in any patient. Congestion and rhinorrhoea reached their maximum value at 40 minutes after L-ASA challenge and lasted up to the end of the study (data not shown). Although one patient with aspirin tolerant asthma/rhinitis developed transient sneezing and congestion 10 minutes after L-ASA instillation, no differences in the clinical response to L-ASA and saline were found either in aspirin tolerant patients or healthy subjects (data not shown).

The relationship between subjective nasal symptoms and changes in nasal volume,
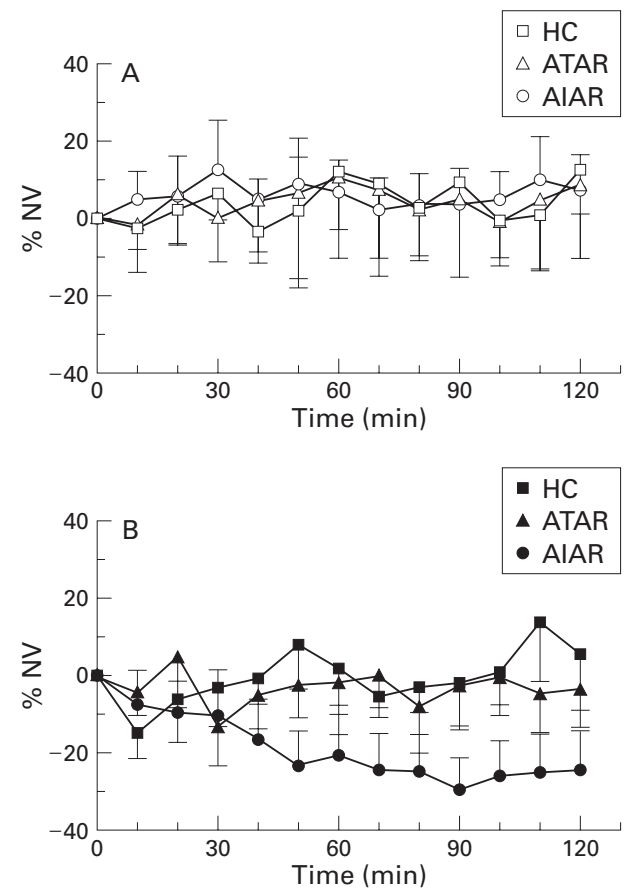

Figure 1 Effects of $(A)$ placebo and $(B) L-A S A$ on percentage change in nasal volume $(\% N V)$ measured by acoustic rhinometry. Values are represented as mean and 95\% confidence intervals. Absence of any superimposition of confidence intervals reveals statistically significant differences. There were no differences in the response to placebo between the three groups. In contrast, a significant decrease in the nasal volume was found at 50 minutes in patients with aspirin intolerant asthmalrhinitis (AIAR) compared with patients with aspirin tolerant

asthma/rhinitis (ATAR) and healthy controls (HC).

expressed as log \%NV, were analysed. Following L-ASA challenge an increase of one unit in the symptom score was associated with a mean (SD) decrease of $2.4(0.3)$ in the $\log \% \mathrm{NV}$ $(\mathrm{p}<0.001)$ in patients with aspirin intolerant asthma/rhinitis. There was no relationship between changes in symptoms and nasal volume with placebo.

The specificity and sensitivity of the nasal challenge in the diagnosis of aspirin sensitive asthma was evaluated by trade off (ROC test). We assessed the sensitivity and specificity of several cut off points in the decrease in log $\% \mathrm{NV}$ (with two consecutive measures under each cut off point). A $25 \%$ decrease in nasal volume was found in at least two consecutive determinations to be the best cut off point. With this percentage decrease the specificity of the test was $94 \%$ and the sensitivity $73 \%$.

Four of the 15 patients with aspirin intolerant asthma/rhinitis had a decrease in nasal volume of $25 \%$ or more following L-ASA challenge. In contrast, only one patient with aspirin tolerant asthma/rhinitis and none of the healthy subjects had a decrease in nasal volume of more than $25 \%$.

The nasal test was well tolerated by all subjects. Aspirin intolerant patients did not experience any clinical deterioration during the procedure. The challenge caused only minor nasal symptoms, similar to those present during spontaneous exacerbations of the disease. Spirometric evaluation was not performed because forced ventilatory manoeuvres 
would have altered the nasal response (personal observation).

\section{Discussion}

This study has shown that nasal challenge with L-ASA is easy to perform and is well tolerated. Nasal provocation with L-ASA induced nasal symptoms almost exclusively in patients with aspirin intolerant asthma/rhinitis, indicating the high specificity of intranasal challenge with L-ASA. None of these patients, some of whom were severely obstructed, developed bronchial symptoms, which suggests that nasal instillation of L-ASA can be used safely in these patients.

We used acoustic rhinometry in the evaluation of the nasal response. This study demonstrates for the first time the effects of nasal congestion precipitated by L-ASA on acoustically determined volumes of the nasal cavity. In aspirin intolerant patients topically administered L-ASA resulted in substantial decreases in the nasal volume. The procedure is very simple and requires minimal time and cooperation from the subjects. The measurements are taken during apnoea ${ }^{10}$ and previous studies have shown very good reproducibility and repeatability. ${ }^{15}$ All these findings suggest that acoustic rhinometry is at least as useful as anterior rhinomanometry in the evaluation of aspirin intolerant asthma/rhinitis.

A spontaneous decrease in the nasal volume was noted while subjects remained seated for the first hour of observation. Although this change was not statistically significant, it should be taken into account when nasal challenges are used for research or diagnostic purposes. A stabilisation period of at least $30 \mathrm{~min}$ utes should be included in the procedure to obtain an adequate baseline value. This initial decrease in nasal volume probably reflects the sensitivity of the nose to changes in position or environmental temperature..$^{16}$

The sensitivity and specificity of several cut off points was assessed and the best cut off point was found to be a fall in the nasal volume of at least $25 \%$ in two consecutive measurements. In our study, however, nasal challenge has a moderate sensitivity in the diagnosis of asthma and rhinitis induced by aspirin. This was probably due, at least in part, to the fact that we used a single dose of L-ASA. A test using progressively increasing doses of L-ASA, as in the inhaled method, might increase the sensitivity of the test but could also increase the potential risk of severe local, bronchial, or systemic reactions. Previous studies have shown that the use of doses of $30 \mathrm{mg}$ or higher of L-ASA cause considerable irritation of the nasal mucosa. ${ }^{68}$ The sensitivity and specificity of our method (using 25\% decrease in nasal volume as the cut off point) was similar to that reported by other authors using rhinomanometry. ${ }^{8} \quad$ Although corticosteroid treatment may reduce the bronchial reactions precipitated by L-ASA in patients with aspirin intolerant asthma, ${ }^{18}$ in our study positive reactions occurred in patients receiving nasal and oral corticosteroids. The doses of nasal and oral corticosteroids were no higher in aspirin intolerant patients with a negative test than in those with a positive response.

We conclude that acoustic rhinometry may be reliably used to study the nasal response after a provocative challenge with L-ASA. Nasal challenge with L-ASA is safe and can be used as a diagnostic test. It might be of use in the study of aspirin sensitivity in patients with severe bronchial obstruction in whom neither the oral nor the inhaled test can be used. Since its use is less limited than anterior rhinometry by the presence of nasal polyps and severe nasal obstruction, acoustic rhinometry can be considered a useful method in the study and diagnosis of aspirin intolerance.

This study was supported by grants from Fondo de Investigaciones Sanitarias (FIS 95-0595) and CIRIT (1998SGR 00112).

1 Szczeklik A, Stevenson DD. Aspirin-induced asthma: advances in pathogenesis and management. $\mathcal{f}$ Allergy Clin advances in pathogenesi

2 Varga EM, Jacobson MR, Masuyama K, et al. Inflammatory cell population and cytokine mRNA expression in the nasal mucosa in aspirin-sensitive rhinitis. Eur Respir $\mathcal{F}$ 1999;14: $610-5$.

3 Nasser SMS, Pfister R, Christie PE, et al. Inflammatory cell populations in bronchial biopsies from aspirin-sensitive asthmatic subjects. Am $\mathcal{F}$ Respir Crit Care Med 1996;153: $90-6$.

4 Sladek K, Dworski R, Soja J, et al. Eicosanoids in bronchoalveolar lavage fluid of aspirin-intolerant patients with asthma after aspirin challenge. Am $\mathcal{f}$ Respir Crit Care Med 1994;149:940-6.

5 Cowburn AS, Sladek K, Soja J, et al. Overexpression of leukotriene $\mathrm{C}_{4}$ synthase in bronchial biopsies from patients with aspirin-intolerant asthma. $\mathcal{F}$ Clin Invest 1998;101:83446.

6 Picado C, Ramis I, Roselló J, et al. Release of peptide leukotriene into nasal secretions after local instillation of aspirin in aspirin-sensitive patients. Am Rev Respir Dis 1992;145: in asp

7 Dahlen B, Zetterström O. Comparison of bronchial and per oral provocation with aspirin in aspirin-sensitive asthmat cs. Eur Respir f 1990;3:527-34.

8 Milewski M, Mastalerz L, Nizankowska E, et al. Nasal provocation test with lysine-aspirin for diagnosis of aspirinsensitive asthma. F Allergy Clin Immunol 1998;101:581-6.

9 Patriarca G, Nucera E, DiRienzo V, et al. Nasal provocation test with lysine acetylsalicylate in aspirin-sensitive patients. Ann Allergy 1991;67:60-2.

10 Fisher EW, Morris DP, Biemans JMA, et al. Practical aspects of acoustic rhinometry: problems and solutions. Rhinology 1995;33:219-33.

11 Grymer LF, Hilberg O, Elbrønd O, et al. Acoustic rhinometry: evaluation of the nasal cavity with septal deviations, before and after septoplasty. Laryngoscope 1989;99: 1180-7.

12 Lenders H, Pirsig W. Diagnostic value of acoustic thinometry: patients with allergic and vasomotor rhinitis compared with normal controls. Rhinology 1990;28:5-16.

13 Lenders H, Schaefer J, Pirsig W. Turbinate hypertrophy in habitual snorers and patients with obstructive sleep apnea: findings of acoustic rhinometry. Laryngoscope 1991;101: 614-8.

14 Hilberg O, Jackson AC, Swift DL, et al. Acoustic rhinometry: evaluation of nasal cavity geometry by acoustic reflection. F Appl Physiol 1989;66:295-303.

15 Castillo JA, Picado C. Prevalence of aspirin-intolerance in a hospital population. Respiration 1986;50:153-7.

16 Lundqvist GR, Pedersen OF, Hilberg O, et al. Nasal reaction to changes in whole body temperature. Acta Otolaryngol (Stockh) 1993;113:783-8.

17 Kase Y, Hilberg O, Pedersen OF. Posture and nasal patency: Kase Y, Hilberg O, Pedersen OF. Posture and nasal patency: evaluation by acoustic

18 Nizankowska E, Szczeklik A. Glucocorticoids attenuate aspirin precipitated adverse reactions in aspirin-intolerant patients with asthma. Ann Allergy 1989;63:157-62. 